\title{
Corneal epithelial recovery following photorefractive keratectomy
}

\author{
Shu-Wen Chang, Fung-Rong Hu, Ping-Kang Hou
}

\begin{abstract}
Aims-To further understand the morphological and functional recovery of corneal epithelium following excimer laser photorefractive keratectomy (PRK).

Methods - The right eyes (group 1) of 15 male, New Zealand white rabbits weighing 2-3 kg underwent PRK. The left eye of each rabbit (group 2) underwent simple mechanical de-epithelialisation and were examined as treated controls. Both eyes of another eight rabbits (group 3 ) served as untreated controls. All eyes underwent a corneal epithelial permeability study by fluorophotometry at 2,4 , and 8 weeks after surgery. Five animals in groups 1 and 2 were sacrificed at 9,10 , and 12 weeks after surgery. The animals in group 3 were sacrificed at the end of the 12 week experimental period. Both eyes of each sacrificed animal were enucleated immediately and processed for both haematoxylin and eosin stain and electron microscopic study. The electron micrograph was magnified to $14000 \times$ and the extent of hemidesmosome formation was quantified and analysed.
\end{abstract}

Results-The corneal epithelial barrier to sodium fluorescein was subnormal and returned to a normal barrier state 4 weeks after PRK in group 1 whereas it was normal in group 2 throughout the examination period. The extent of hemidesmosome formation was abundant yet subnormal in both groups 1 and 2 up to 12 weeks, when compared with that in group 3 .

Conclusion-The corneal epithelium regained its functional barrier 4 weeks after PRK in rabbits while the extent of hemidesmosome formation was still subnormal 12 weeks after mechanical deepithelialisation, with or without PRK. (Br f Ophthalmol 1996;80:663-668)

Department of Ophthalmology, National Taiwan University Hospital, 7 Chung-Shan S Road, Taipei, Taiwan, ROC

S-W Chang

F-R Hu

P-K Hou

Correspondence to:

Dr Shu-Wen Chang.

Accepted for publication 15 April 1996
Excimer laser photorefractive keratectomy (PRK) is becoming popular for the correction of myopia. The success of this procedure is usually appraised by the absence of subepithelial haze, the visual results, postoperative residual refractive errors, and long term stability. Although the corneal epithelium might appear normal under slit-lamp microscopic examination after PRK, it remains unclear if there is a subclinical alteration. Since the relative impermeability of corneal epithelium is crucial to maintain the homeostasis of fluid and solutes between the intraocular milieu and precorneal tear film, the restoration of both normal histological arrangement and functional/physiological corneal variables must be demonstrated before justifying the long term safety of this procedure in an otherwise normal eye.

Previous morphological research on the PRK wound is extensive. Histologically, the corneal epithelium increased its thickness by $50 \%$ in the early postoperative period, and attached itself to the underlying stroma with formation of basal lamina and hemidesmosomes following PRK. ${ }^{1-5}$ However, the epithelial barrier study has not been performed. It had been demonstrated that the status of corneal epithelium can influence the connective tissue synthesis after keratectomy, ${ }^{6}$ which subsequently results in corneal haze and a change in the desired refraction. ${ }^{56}$ Although it was claimed that the corneas healed satisfactorily following PRK, simple epithelial removal might cause loss of superficial stromal keratocytes in rabbits and monkeys. ${ }^{8}$ Also, despite the fact that more than $80 \%$ of the PRK cases showed normal epithelium, observed by specular microscope clinically suggesting that the absence of Bowman's layer produced by PRK might not have a significant effect on epithelial morphology, ${ }^{9}{ }^{10}$ there was a mild decrease in corneal sensitivity in the first 6 weeks after surgery. ${ }^{11}$ Because sensory denervation of the cornea results in a decreased mitotic activity, ${ }^{12} 13$ impaired epithelial healing process, ${ }^{14}$ and impaired cellular adhesion ${ }^{15}{ }^{16}$ in the epithelial cells, understanding the restoration of the epithelial barrier function after PRK is helpful in assessing its safety.

\section{Materials and methods}

Fifteen male, New Zealand white rabbits weighing approximately $2-3 \mathrm{~kg}$ were maintained in an accredited facility according to Association for Research in Vision and Ophthalmology standards for the use of animals. The right eye of each animal underwent PRK (group 1), while the left eye of each rabbit underwent mechanical de-epithelialisation without PRK (group 2).

EPITHELIAL PERMEABILITY

A volume of $20 \mu \mathrm{l}$ of $2 \%$ sodium fluorescein solution was instilled into both conjunctival cul de sacs of each animal. They were anaesthetised with intramuscular ketamine and xylazine (3:2) 45 minutes after topical instillation of the fluorescein solution, after which corneal fluorescein concentration was measured with a fluorophotometer (Fluorotron Master-2). Four fluorescein concentrations at the corneal peak 
Table 1 Summary of corneal fluorescein concentrations at different times after photorefractive keratectomy

\begin{tabular}{lllll}
\hline & Group 1 $(n=15)$ & Group 2 $(n=15)$ & Group 3 $(n=16)$ & pValue \\
\hline Week 2 & $988.8(217.7)^{*}$ & $324.4(29.2)$ & $306.4(23.2)$ & $<0.005$ \\
Week 4 & $320.1(39.2)$ & $300.6(50.8)$ & $267.4(20.3)$ & NS \\
Week 8 & $397.0(66.5)$ & $364.1(71.0)$ & $271.1(20.6)$ & NS \\
\hline
\end{tabular}

Corneal fluorescein concentrations $\mathbf{4 5}$ minutes after topical fluorescein application (F45) (ng/ml). Numbers represent mean (SE). p Value=statistical significance examined by one way analysis of variance.

*Statistically significant difference ( $p<0.05$ examined by Tukey multiple comparison test) when compared with groups 2 and 3 . NS=not significant.

were averaged from each scan to represent the corneal epithelial permeability (F45).

\section{PHOTOREFRACTIVE KERATECTOMY}

The animals were anaesthetised with intramuscular ketamine and xylazine (3:2). The central corneal epithelium in both eyes of each rabbit was debrided mechanically by a No 64 Beaver (Waltham, MA, USA) blade. The right eye was then positioned under the laser, using the pupil as the centre for ablations. A $193 \mathrm{~nm}$ argon fluoride excimer laser (Summit) was used in these experiments. The laser was programmed to perform myopic ablations of 6 dioptres (D) with no cylindrical change. For all ablations, the average fluence was $180 \mathrm{~mJ} / \mathrm{cm}^{2}$ with a firing rate of $10 \mathrm{~Hz}$ and an ablation zone diameter of $6.0 \mathrm{~mm}$. At the completion of photoablation, topical ciprofloxacine eyedrops were instilled into both lower conjunctival sacs. Corneal re-epithelialisation was complete within 7 days.

Both eyes of each rabbit in groups 1 and 2 received corneal epithelial permeability examination at 2,4 , and 8 weeks after PRK. The same examination was performed in parallel on another eight male, New Zealand white rabbits to serve as a control group (group 3). However, all 16 corneas of these rabbits in group 3 were not mechanically debrided.

The difference of F45s between the right (group 1) and left (group 2) eyes were examined by paired $t$ test. The difference of F45s among all three groups was examined by one way analysis of variance (ANOVA). When a difference reached a statistical significance of $\mathrm{p}<0.05$, it was further tested by the Tukey multiple comparison test.

\section{MORPHOLOGICAL EXAMINATION}

Five animals in groups 1 and 2 were sacrificed at 9,10 , and 12 weeks following PRK for morphological study. All eight animals in group 3 were sacrificed at the end of 12 weeks. Both eyes of the animals were enucleated immediately following an intravenous overdose of pentobarbitone. Ten per cent formaldehyde solution was injected into the anterior chamber and vitreous cavity. The whole eyeball was then immersed in $10 \%$ formaldehyde for $30 \mathrm{~min}$ utes. A block of central cornea, measured $1 \times 1$ $\times 0.4 \mathrm{~mm}$ in size, was excised and immersed in $2.5 \%$ glutaraldehyde solution. The rest of the corneal tissue was further processed for haematoxylin and eosin stain and observed by light microscope. The excised tissue block was postfixed in $1 \%$ osmium tetroxide, and embedded in Spurr resin medium. Thin sections were stained with uranyl acetate-lead citrate and examined with a transmission electron microscope. To estimate the extent of hemidesmosome formation, an area of basal corneal epithelium and superficial stroma was photographed at $7000 \times$. It was then printed at a final magnification of $14000 \times$. The photographs were masked. The length of basal cell membrane covered by hemidesmosomes (HE) and the length of the basal cell membrane (BM) were measured using a $10 \times$ magnifier to facilitate viewing. The percentage of basal cell membrane covered by hemidesmosome was calculated by $(\mathrm{HE} / \mathrm{BM}) \times 100$ and called the hemidesmosome index (HI). Three photographs were measured and averaged, measuring about $120 \mu \mathrm{m}$ of basal cell membrane in each eye. The HI of groups 1 and 2 at 9,10 , and 12 weeks following PRK were compared using the paired $t$ test. The difference in HI among all three groups was examined by one way analysis of variance (ANOVA). Because the rabbits in group 3 were not examined for $\mathrm{HI}$ at 9 and 10 weeks, data obtained at 12 weeks were used for analysis. When a difference reached a statistical significance of $p<0.05$, it was further tested by the Tukey multiple comparison test.

\section{Results}

The F45s of all three groups at different intervals following PRK are summarised in Table 1. The F45s in group 1 was significantly higher than those in groups 2 and $3(p<0.01$ and $\mathrm{p}<0.005$ respectively) in the early postoperative period. However, the averaged epithelial barrier recovered to the baseline level 1 month after PRK. The barrier function in group 2 did not differ from that in group 3 throughout the examination periods.

On histological examination, the corneal epithelium thickened with both a vertical elongation of the basal cells and an increase in the number of the superficial cell layers. There was a zone of increased keratocyte density in the superficial stroma (Fig 1). However, the epithelium regained its normal thickness and the superficial stromal keratocyte density decreased 12 weeks postoperatively. Under the electron microscope, there were tight junctions between the superficial epithelium cells in all corneas from 9 to 12 weeks. The cell membrane of the basal corneal epithelium fac-

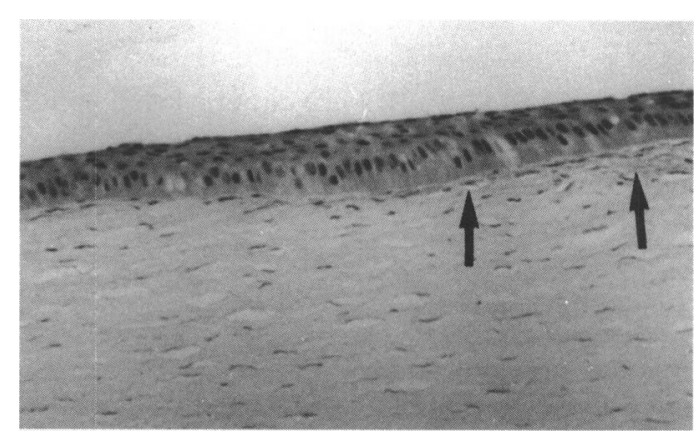

Figure 1 Section of rabbit central cornea 9 weeks after photorefractive keratectomy. The corneal epithelium was hyperplastic and there was a zone of increased density of superficial stromal keratocyte (arrows) (haematoxylin and eosin, $\times 200$ ). 
ing corneal stroma was slightly deranged with a focal increase of intercellular interdigitation up to 12 weeks postoperatively in group 1 (Fig 2) when compared with that of control eyes (Fig 3). The basal cell membrane in group 2 remained normal throughout the experiment.

The hemidesmosome index (\%) in group 1 (mean (SE)) was 25.2 (4.9), 28.2 (1.6), and $29.3(1.1)$ at 9,10 , and 12 weeks, respectively. Although there was a tendency towards more hemidesmosome formation from the ninth to the 12th week, the trend was not statistically significant. The HI in group 2 was 27.4 (4.2), $31.4(3.9)$, and $36.0(1.0)$ at 9,10 , and 12 weeks, respectively. Corneas at 12 weeks after mechanical de-epithelialisation had slightly higher hemidesmosome index when compared with that at ninth week $(p=0.05)$. The

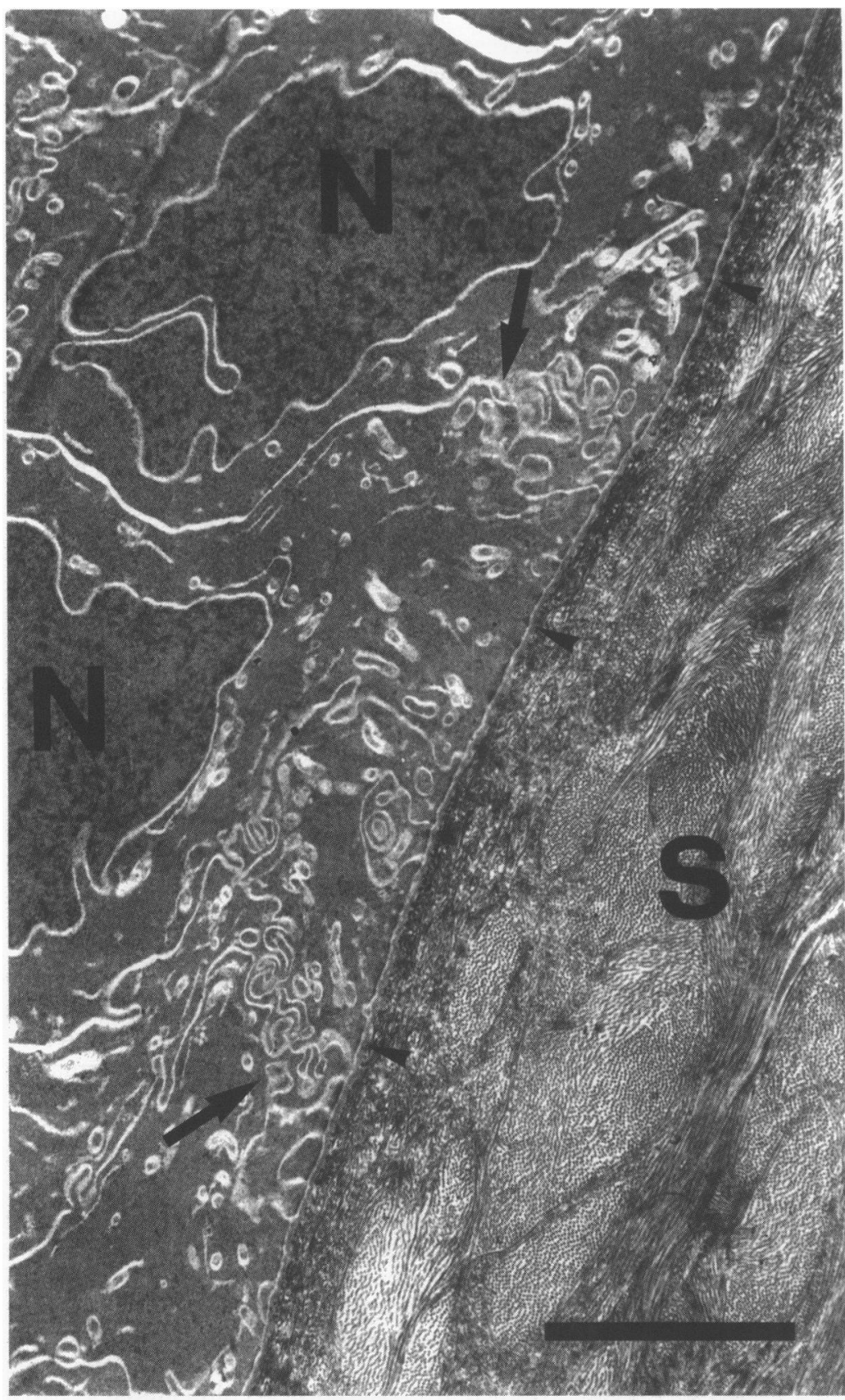

Figure 2 Electron micrograph of the basal corneal epithelium 12 weeks after

photorefractive keratectomy. The cell membrane of basal corneal epithelium was deranged with a focal increase in intercellular interdigitation (arrows). The hemidesmosomes were less in both numbers and extent (arrowheads). $N$, nucleus of basal corneal epithelium; $S$, superficial stromal fibrils. Bar=10 $\mu \mathrm{m}$. hemidesmosome index of group 3 was 49.4 (1.7) at 12 weeks. The hemidesmosome index was significantly more in group 3 when compared with those of groups 1 and 2 at all intervals $(\mathrm{p}<0.05)$. The hemidesmosome index was more in group 2 when compared with that in group 1 at 10 and 12 weeks $(p<0.005)$.

In group 1, there was a subepithelial zone of deranged collagen deposition. This zone measured $2.8-3.3 \mu \mathrm{m}$ in depth at 9 weeks and decreased to $2.0-2.5 \mu \mathrm{m}$ at 12 weeks.

\section{Discussion}

The corneal epithelium maintains an ideal barrier from the surrounding milieu. To obtain this, the basal corneal epithelium anchors itself to the underlying tissue by forming an adhesion complex and the superficial epithelial cells maintain this barrier function by their cell membranes and intercellular tight junctions. To understand the epithelial recovery following PRK, morphological and functional studies on both intercellular tight junctions and hemidesmosomes is warranted. In this study, tight junctions were seen between the superficial cells in all specimens obtained from 9 to 12 weeks following PRK. However, a quantitative morphological study on tight junctions was difficult and thus not performed. Instead, we used water soluble sodium fluorescein topically, which permeated into the corneal stroma mainly through the intercellular route, and evaluated the functional integrity of the intercellular tight junctions. The epithelial barrier tested by topical instillation of sodium fluorescein is valuable in detecting epithelial barrier function abnormalities in diabetic, post penetrating keratoplasty and aging corneas. ${ }^{17-20}$ Because there was no functional study of the hemidesmosomes available, we adopted a quantitative morphological study of the hemidesmosome index to evaluate its recovery following PRK.

After PRK, the corneal epithelium had an abnormal barrier function to topically applied sodium fluorescein. It recovered to the baseline barrier function 1 month later. In the first month after PRK, there was a relatively large interindividual variation in F45s. This might be attributed to the diversity in the recovery rate of tight junction function among individuals since all corneas had complete reepithelialisation by the first postoperative week. However, we were unable to exclude the possibility that undetected recurrent corneal erosion developed during the follow up pe$\operatorname{riod}^{21}$ and the newly regenerated corneal epithelium thus had a subnormal barrier function in the first month. Since a small central epithelial defect in four of 21 eyes was reported after initial re-epithelialisation following mechanical keratectomy, ${ }^{22}$ we examined the epithelial permeability for a longer period after laser ablation and found no abnormality in the barrier function 8 weeks postoperatively. In corneas that underwent simple mechanical de-epithelialisation, there was no significant breakdown in the epithelial barrier function when compared with that of control eyes. 
Because there was marked interdigitation of epithelial cell membrane, a quantitative study on the tight junction formation was difficult and thus was not performed here. Nevertheless, we assumed there was sound intercellular tight junction formation during the first month following PRK since the corneal epithelial barrier function was competent in 4 weeks. The reasons for a subnormal epithelial barrier function in the absence of an epithelial defect is obscure. We proposed that corneal denervation during the PRK procedures might play a role because the corneal sensory nerves can have a trophic influence on the corneal epithelium. In human eyes, corneal hypaesthesia was noticed in the first 6 weeks following PRK. ${ }^{11}$ It is possible that sensory denervation of the cornea in the early stage following PRK resulted in impaired epithelial healing, impaired cellular adhesion $^{13-16}$ and also decreased barrier function in spite of there being no corneal epithelial

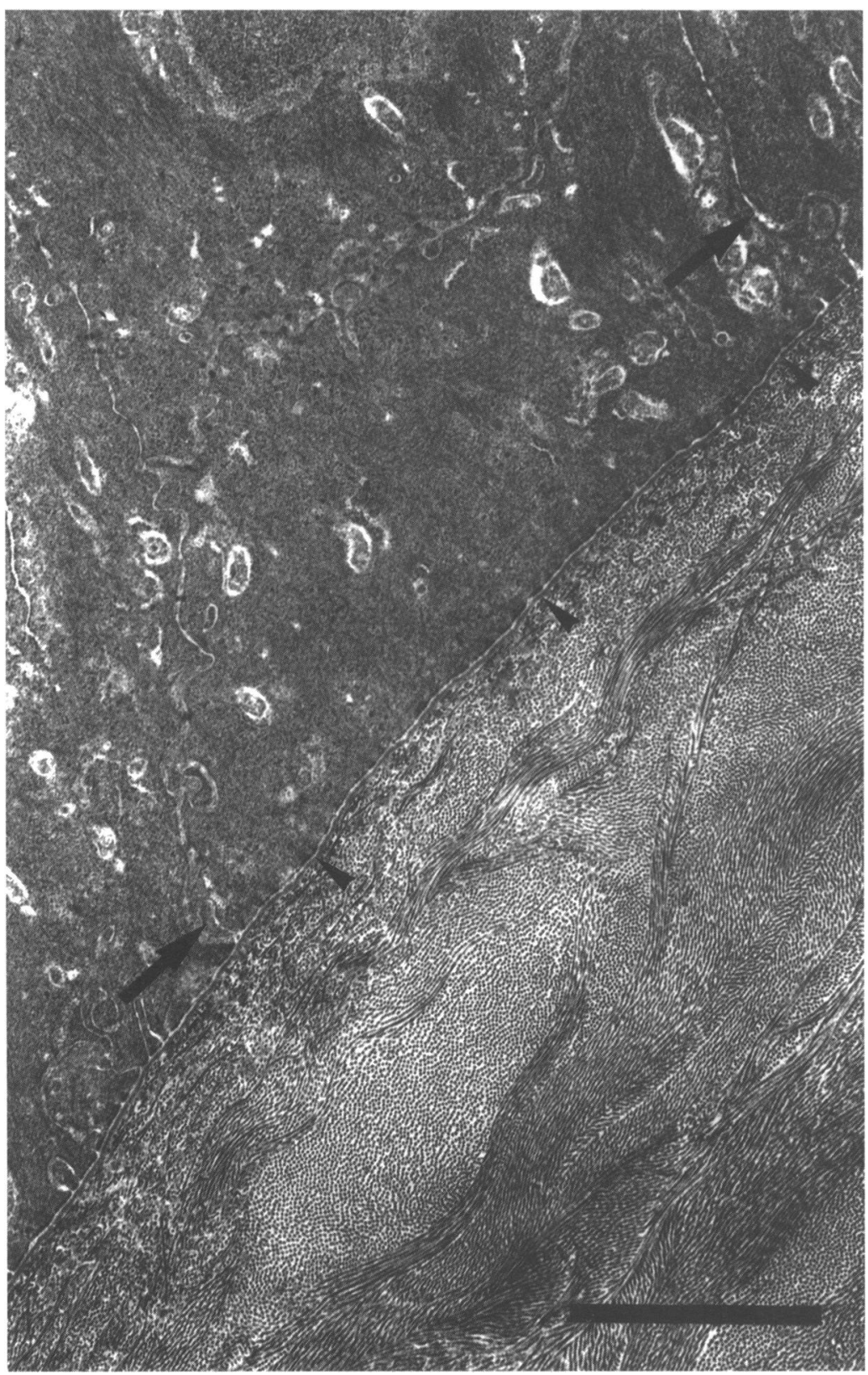

Figure 3 Electron micrograph of the basal corneal epithelium in group 3. The cell membrane of the basal corneal epithelium was smooth (arrows) and the hemidesmosomes were numerous and more in extent (arrowheads). Bar $=10 \mu \mathrm{m}$. defect. This condition is also documented in diabetic and post penetrating keratoplasty corneas, which are accompanied by corneal hypaesthesia. ${ }^{1819}$ The abnormal epithelial cell morphology in the early postoperative period may also contribute partially to the subnormal barrier function..$^{10}$ In group 2, although the corneal epithelium was mechanically deepithelialised, the epithelial barrier function was normal after re-epithelialisation because simple mechanical de-epithelialisation did not lead to corneal hypaesthesia. It is possible that subnormal epithelial barrier function in human eyes lasts longer after PRK because the rabbit cornea does not have a Bowman's layer and has a more rapid healing course following PRK..$^{23} 24$ The clinical significance of this early postoperative subnormal epithelial barrier function following PRK remains unknown; however, the corneal surgeons should be cognizant of this decrease in corneal epithelial barrier function in the early postoperative period and topical medication during this period should be given carefully to minimise its cumulative effect on the corneal stromal keratocytes and/or endothelial cells in the presence of such a subnormal epithelial barrier function.

On histological examination, there was an initial thickening of the epithelium over the bed of anterior keratectomy specimens with both a vertical elongation of the basal cells and an increase in the number of the superficial cell layers, which attenuated 12 weeks postoperatively. This finding was comparable with previous studies, ${ }^{4-6}{ }^{25}$ in which the corneal epithelium regained its normal thickness 3-6 weeks or even longer after PRK, according to different experimental designs. The zone of deranged subepithelial collagen fibrils decreased 12 weeks postoperatively in this study corresponding to the previous observation that the subepithelial collagen became more organised with time. ${ }^{5}$ However, there was an interindividual variation in recovery because excimer lasers commonly have variations in pulse energy during a single ablation and from one animal to the next, although all efforts have been made to limit these compounding factors to as minimal as possible. ${ }^{4}$

On the side facing the corneal stroma, the cell membrane of the basal epithelium was slightly deranged with a focal increase of intercellular interdigitation under the electron microscope up to the 12 th postoperative weeks in group 1, whereas it remained normal in group 2 . It is possible that the slightly uneven superficial stroma or the changes in the superficial stroma following PRK rendered the regrown basal cell membrane irregular.

In this study, it is not determined whether there is a difference in the extent of hemidesmosome formation between groups 1 and 2 in the early postoperative period since morphological evaluation was not performed in this period. However, the electron microscopic study of the basal corneal epithelium revealed a subnormal extent of hemidesmosome formation up to 12 weeks in both groups, although they seemed abundant in appearance as in previous reports. ${ }^{14}$ According to previous re- 
search, when basement membrane is damaged experimentally by any technique, which results in discontinuities and duplications of basement membrane complexes, the epithelium is easily separable from the stroma for 8 weeks or more. ${ }^{26-28}$ The corneal epithelial basement membrane was removed during PRK and probably damaged during mechanical deepithelialisation in this study, which might contribute partially to the defective hemidesmosome formation in groups 1 and 2 and remained subnormal in extent up to 12 weeks after de-epithelialisation with or without PRK. In group 1 , the early postoperative corneal hypaesthesia, superficial irregularity, and scarring following stromal removal might further retard the formation of hemidesmosomes. Besides, in the presence of a subnormal extent of hemidesmosome formation, the basal epithelial cells further increased their intercellular interdigitation in compensation, attempting to achieve a firmer adhesion to the remaining stroma following PRK. It seems apparent that an increase in intercellular interdigitation leads to increased intercellular adhesion but not to increased basal adhesion to underlying stroma. However, if some cells have firmer adhesion to the underlying stroma than the others-for example, those at the untreated area or some focally better healed points, increased intercellular adhesion may indirectly increase the entire epithelial sheet's adhesion to the underlying stroma.

Because reduced hemidesmosome density correlated with a reduced epithelial adhesion, ${ }^{29}$ it is possible that abnormal hemidesmosome formation may lead to recurrent epithelial breakdown and subsequent corneal erosion. ${ }^{21}$ On the other hand, unhealthy corneal epithelial cell per se-that is, a defective cell membrane, inadequate cell alignment, ${ }^{9} 10$ and poor intercellular tight junction may both result in a subnormal barrier function. In this study, there was statistically significantly less hemidesmosome formation up to 12 weeks in corneas after mechanical de-epithelialisation, with or without PRK. The observed reduction in hemidesmosomes indicates a compromise of the epithelial adhesion complex since the assembly of hemidesmosomes, basement membrane, and anchoring fibrils tends to occur synchronously in corneal wound healing. ${ }^{22}$ It is true that a decreased extent of hemidesmosome formation does not necessarily lead to a manifest corneal epithelial breakdown and in fact no episodes of corneal erosion were noticed in this experiment and in Gipson's series for up to 1 year of follow up. The existence of subnormal hemidesmosome formation, however, may indicate a potential of having an epithelial problem in response to additional trauma. Although it is not known to what extent will the decrease in hemidesmosome formation cause clinically significant corneal erosion, we believe that this abnormality contributed partially to tenderness on eye rubbing, foreign body sensation on awaking, and epithelial instability in some cases in the postoperative period. ${ }^{21}$

Although refractive surgery has been used commonly to correct ametropia, the predict- ability of existing keratorefractive procedures was weakened by the variability in corneal biomechanical properties and wound healing processes among individuals. In this study, we compared the difference in the functional and morphological recovery following simple mechanical epithelial removal and photorefractive keratectomy in both eyes of each animal, attempting to reduce the interindividual variation. Besides, both eyes of each animal in group 3 were examined as a control to reduce the intraindividual variation in methodology. We demonstrated a subnormal corneal epithelial barrier function in the eyes 1 month after PRK and subnormal hemidesmosome formation 3 months after PRK. We believe this may contribute to the small number of patients who have either a foreign body sensation, watering, or tenderness on eye rubbing up to 2 years after surgery. ${ }^{21}$ Further long term studies are required to appraise its clinical significance.

1 Taylor DM, L'Esperance FA Jr, Del Pero RA, Roberts AD, Gigstad JE, Klintworth KG, et al. Human excimer laser lamellar keratectomy. A clinical study. Ophthalmology 1989;96:654-64.

2 McDonald MB, Frantz JM, Klyce SD, Salmeron B, Beuerman RW, Munnerlyn CR, et al. One-year refractive results of central photorefractive keratectomy for myopia in the nonhuman primate cornea. Arch Ophthalmol 1990;108: 40-7.

3 Gauthier CA, Holden BA, Lawless MA, Rogers CM. Epithelial alterations following photorefractive keratectomy for myopia. Invest OphthalmolVis Sci 1993;34(suppl):798.

4 Hanna KD, Pouliquen Y, Waring III GO, Savoldelli M, Cotter J, Morton $\mathrm{K}$, et al. Corneal stromal wound healing in rabbits after 193-nm excimer laser surface ablation. Arch Ophthalmol 1989;107:895-901.

5 Del Pero RA, Gigstad JE, Roberts AD, Klintworth GK Martin CA, L'Esperance FA, et al. A refractive and histopathologic study of excimer laser keratectomy in primates. Am ₹ Ophthalmol 1990;109:419-29.

6 Tuft SJ, Zabel RW, Marshall J. Corneal repair following keratectomy. A comparison between conventional surgery and laser photoablation. Invest Ophthalmol Vis Sci 1989;30: and laser pho

7 Dunnington JH, Weimar V. Influence of the epithelium on the healing of corneal incisions. Am $f$ Ophthalmol 1958;45(4, part II):89-95.

8 Szerenyi K, Campos M, Raman S, Lee M, MaDonnel J, McDonnell PJ. Keratocyte loss after corneal deepithelialization in primates and rabbits. Invest Ophthalmol Vis Sci 1993;34(suppl):802.

9 Amano S, Shimizu K, Tsubota K, Nouka K. Corneal epithelial changes after excimer laser photorefractive keratectomy. Am $\mathcal{f}$ Ophthalmol 1993;115:441-3.

10 Amano S, Shimizu K, Tsubota K. Specular microscopic evaluation of the corneal epithelium after excimer laser photorefractive keratectomy. Am $₹$ Ophthalmol 1994;117: photore.

11 Campos M, Hertzog L, Garbus JJ, McDonnell PJ. Corneal sensitivity after photorefractive keratectomy. Am $\mathcal{F}$ Ophthalmol 1992;114:51-4.

12 Mishima S. The effects of the denervation and the stimulation of the sympathetic and the trigeminal nerve on the mitotic rate of the corneal epithelium in the rabbit. $\mathcal{f p n} \mathfrak{F}$ Ophthalmol 1957;1:65-73.

13 Sigelman S, Friedenwald JS. Mitotic and wound-healing activities of the corneal epithelium; effect of sensory denervation. Arch Ophthalmol 1954;52:46-57.

14 Beuerman RW, Schimmelpfenning B, Buerstein N. Sensory denervation of the rabbit cornea affects epithelial properties. Exp Neurol 1980;6:196-201.

15 Beuerman RW, Schimmelpfenning B, Burstein N. Anatomy of the denervated corneal epithelium. ARVO Abstracts. Invest OphthalmolVis $S c i$ 1979;18(suppl): 126 .

16 Araki K, Ohashi Y, Kinoshita S, Hayashi K, Kuwayama Y, Tano Y. Epithelial wound healing in the denervated cornea. Tano Y. Epithelial wound healing

17 de Kruijf EJ, Boot JP, Laterveer L, van Best JA, Ramselaar JA, Oosterhuis JA. A simple method for determination of corneal epithelial permeability in humans. Curr Eye Res 1987;6:1327-34.

18 Chang SW, Hsu HC, Hu FR, Chen MS. Corneal autofluorescence and epithelial barrier function in diabetic patients. Ophthalmic Res 1995;27:74-9.

19 Chang SW, Hu FR. Corneal epithelial barrier function following penetrating keratoplasty. Ophthalmic Res 1994; 26:283-9.

20 Chang SW, Hu FR. Changes in corneal autofluorescence and corneal epithelial barrier function with ging. Cornea 1993;12:493-9. 
21 Gartry DS, Kerr Muir MG, Marshall J. Excimer laser photorefractive keratectomy: 18 month follow-up. Ophthalmoogy 1992;99:1209-19.

22 Gipson IK, Spurr-Michaud S, Tisdale A, Keough M. Reassembly of the anchoring structures of the corneal epithelium during wound repair in the rabbit. Invest Ophthalmol lium during wound repair

23 Marshall J, Trokel S, Rothery S, Krueger RR. Photoablative reprofiling of the cornea using an excimer laser: photore fractive keratectomy. Lasers Ophthalmol 1986;1:21-48.

24 Tuft S, Marshall J, Rothery S. Stromal remodeling following photorefractive keratectomy. Lasers Ophthalmol 1987;1: $177-83$.

25 Beuerman RW, McDonald MB, Shofner RS, Munnerlyn histological studies of primate corneas after excimer laser photorefractive keratoplasty. Arch Ophthalmol 1994;112: $1103-10$

26 Folge JA, Neufeld RH, Stark WJ. Damage to epithelial basement membrane by thermokeratoplasty. Am f Ophthalmol 1977;83:392-401.

27 Kenyon KR, Folgle JA, Stone DL, StarkWJ. Regeneration of corneal epithelial basement membrane following thermal corneal epithelial basement membrane following thermal 292-301.

28 Khodadoust AA, Silverstein AM, Kenyon KR, Dowling JE Adhesion of regenerating corneal epithelium: The role of basement membrane. Am $f$ Ophthalmol 1968;65:339-48.

29 Madigon MC, Holden BA. Reduced epithelial adhesion after extended contact lens wear correlates with reduced hemidesmosome density in cat. Invest Ophthalmol Vis $\mathrm{Sci}$ 1992;33:314-23. 